\title{
Proliferation of Hemopoietic Colony-Forming Cells Transplanted into Irradiated Mice'
}

\author{
E. A. McCULLOCH AND J. E. TILL \\ Department of Medical Biophysics, University of Toronto, and The Ontario Cancer \\ Institute, Toronto, Ontario
}

\section{INTRODUCTION}

It is generally agreed that survival of mice after whole-body irradiation depends on the restoration of hemopoietic function. This restoration may result from the proliferative activity of cells that have survived the radiation, or of cells from unirradiated donors that have been transplanted into the irradiated animals. Recently, we have suggested that such hemopoietic restoration depends in large measure on the activity of a class of cells that can be recognized by their ability to give rise to cellular colonies in the spleens of irradiated mice (1). The evidence relating colony-forming ability to survival of the animal is as follows: First, analysis of the cellular composition of colonies shows that they include both new colony-forming cells (2) and more differentiated erythrocytic, myelocytic, and megakaryocytic elements ( 9 ); thus colony-forming cells have the capacity for self-renewal which is necessary for continuing hemopoietic function, and the capacity to give rise to differentiated cells which are necessary for the survival of the animal. Second, the kinetics of repair of damage to colony-forming cells between fractionated doses of radiation is very similar to the kinetics of repair of radiation injury leading to death (1). This similarity supports the suggestion that an increase in the number of cells with colony-forming ability may form an important part of the cellular basis of repair of radiation damage where death is the criterion of injury.

After sublethal irradiation, the restoration process in hemopoietic tissue may be considered to consist of two major phases, "early" and "late" repair (1). Early repair, which occurs during the first day after irradiation, is probably primarily the result of an intracellular process of recovery which takes place in sublethally damaged cells, in the absence of cellular proliferation. This recovery process has

1 This research was supported by the Defence Research Board of Canada (Grant 9350-14 G\&C), by the National Research Council of Canada (A 1376), by the National Cancer Institute of Canada, and by the Medical Research Council of Canada (MA-1420). 
been studied in detail in mammalian cell cultures by Elkind and Sutton (4). Late repair, occurring after the first day postirradiation, appears to be primarily the result of cellular proliferation which leads to the production of new hemopoietic cells (1). In a previous study (5) of the early repair process in spleen colony-forming cells, it was not possible to establish with certainty that the initial maximum in early repair observed 5 hours after irradiation was the result of recovery of the type described by Elkind and Sutton, since it was not shown whether or not cellular proliferation was occurring at this time. Thus, in order to make a distinction between this type of recovery and other forms of repair, it is necessary to obtain a knowledge of the growth kinetics of the cells involved. In the experiments reported in this paper, two methods were used to measure the growth kinetics of normal colony-forming cells after transplantation into irradiated hosts. The results indicate that little or no proliferation occurs for the first day after transplantation and that this lag is followed by a period of exponential growth with a doubling time of 20 to 25 hours. Thus, the early repair observed in the previous study (5), which was carried out during the first day after transplantation, must have been due to recovery of the Elkind type.

\section{MATERIALS AND METHODS}

\section{Mice}

The mice used in these experiments were bred in the breeding colonies of the Ontario Cancer Institute. The inbred animals were of strains $\mathrm{C} 3 \mathrm{H}$ and C57BL, and the F1 hybrid between them. Complete designations of these animats have been published $(6)$. In addition, randomly bred Swiss mice were used in some experiments. After irradiation and marrow transplantation the mice were housed 2 to 4 animals per cage and allowed food and water freely.

\section{Irradiation Techniques}

The irradiation procedures were similar to those previously described (7). Irradiation of recipient mice was carried out with 280-kvp X-rays, HVL $1.12 \mathrm{~mm} \mathrm{Cu}$, at an exposure rate of approximately $100 \mathrm{r} / \mathrm{min}$. Marrow cell irradiations were carried out with $\mathrm{Co}^{60} \gamma$-rays delivered at a dose rate of about $50 \mathrm{rads} / \mathrm{min}$. These irradiations were performed in vivo on groups of 10 mice in a circular Lucite holder $20 \mathrm{~cm}$ in diameter, placed on a revolving turntable $50 \mathrm{~cm}$ from the $\mathrm{Co}^{60}$ source. Dosimetry was based on measurements of exposure with scatter, made with a Victoreen r-meter, and on absorbed dose measurements made with the ferrous sulfate dosimeter (8). A $G$ value of 15.5 ferric ions per 100 ev was assumed. Dose measurements were made in the Lucite holder at a point corresponding to that of a representative animal, with the dosimeter surrounded by wax to simulate the conditions existing during irradiation of the mice. 


\section{Measurements of Colony Formation}

The methods used for measurement of colony formation by transplanted marrow cells in the spleens of irradiated animals have been described in detail $(7,9)$. It should be noted that an unavoidable dilution of a cell suspension occurs when it is tested for colony-forming ability by injection into an irradiated recipient mouse, in that only a fraction, $f$, of the injected colony-forming cells actually lodge in the spleen and there form colonies. Such cells are termed colony-forming units (CFU). For cells derived from marrow, the value of $f$ has been found to be 0.17 (2).

\section{Measurement of Cell Proliferation by Periodic Sampling}

The method of periodic sampling used initially for measurement of the growth curve of colony-forming cells was as follows: A known number of marrow cells was injected into approximately 100 irradiated (900 rads) recipient anaimals. At various times thereafter, groups of 10 or more animals were killed, and their spleens were removed and dispersed to form a single cell suspension (6). The cell suspensions thus obtained were then tested for their content of CFU by injection of a known proportion of the suspension into irradiated recipient mice. From the resulting number of colonies, it was possible to calculate the total number of CFU recovered from the spleens as a function of time after transplantation. The accuracy of the growth curves obtained by this method is dependent on the efficiency with which CFU may be recovered from the spleens of the irradiated recipient mice.

\section{Radiobiological Measurement of Cell Proliferation}

The radiobiological technique used as an alternative means of studying the proliferation of colony-forming cells represents an extension of the method usually used for measuring survival of the colony-forming capacity of cells irradiated in vivo (7). In brief, this method was as follows: Groups of mice were irradiated with a portion of the large dose (900 rads or more) required to prepare them as recipients of marrow cells. Then an appropriate number of nucleated cells from the femoral marrow of donor mice was injected intravenously. At various times thereafter, a second dose of radiation, of $\mathrm{Co}^{60} \gamma$-rays, was given. Ten days after the second dose, the animals were killed, and the mean number of colonies per spleen in each group was determined. The two radiation doses were so arranged that their sum was equal to a dose sufficient to reduce colony formation from endogenous colony-forming cells to less than an average of 0.5 colony per mouse (5). The total dose required for this purpose was determined from experiments in which the effects of dose fractionation on the survival of endogenous colony-forming cells were studied (1). Whereas the cells of the recipient animal received this total dose, the grafted marrow cells received only the radiation delivered in the second exposure, and the survival of their colony-forming ability was a function only of this dose. 
In these experiments, the shortest interval between injection of the cells and the second irradiation was 2 hours. During this short interval of time, cell proliferation may be assumed to have been negligible. When this interval was prolonged for a time long enough to permit cell proliferation to occur, however, one would expect the resulting increase to be reflected in a change in the survival curve obtained when the in vivo irradiation was done after the prolonged interval. This survival curve may be assumed to have the form

$$
S=1-\left(1-e^{-D / D_{0}}\right)^{n}
$$

where $S$ is the fractional survival observed after a single dose $D, D_{0}$ is the recip rocal of the magnitude of the slope of the survival curve, measured on the exponen ${ }^{-}$ tial portion of the curve, and $n$ is the extrapolation number. If cells in close proximity are inactivated independently, so that the condition of "survival independence" is fulfilled, and provided that the distribution of probabilities of survival for the population of individual colony-forming cells does not change with time (owing, for example, to a change in the distribution of cell numbers in different portions of the division cycle, (10), then an increase in the number of colonyforming cells should cause a change only in the extrapolation number of the survival curve and not in its slope (see, for example, the work of Elkind and co-workers, $(4,11)$. Further, if the above conditions are met, it would be expected that, for the exponential portion of the survival curve, the factor by which the per cent survival increased in the interval when cellular proliferation occurred should equal the factor by which the mean number of colony-forming cells increased. This factor has been termed the "cellular multiplicity" (4). Thus, if only fractional survivals on the exponential portion of the survival curve are considered, one would expect the relation shown in equation 1 to be applicable. Here $S_{0}$ represents the

$$
R(t)=S(t) / S_{0} \simeq m(t)
$$

fractional survival observed when the cells are irradiated with a dose $D$ shortly after transplantation, and $S(t)$ represents the fractional survival obtained when the dose $D$ is delivered after a time interval $t$ has elapsed; the ratio of these fractional survivals, $R(t)$, is the "survival ratio," and $m(t)$ is the cellular multiplicity. As has been pointed out by Elkind and Sutton, expression 1 should be a good approximation for fractional survivals of less than 0.1 .

Thus, if the above conditions are met, a series of measurements of the survival ratio, as a function of time after irradiation, should provide a simple means of following the proliferation of colony-forming cells. The technique is analogous to that used by Luria and Latarjet (12) in an attempt to study the intracellular development of bacteriophage particles. 


\section{RESULTS}

\section{Growth Curve Obtained by the Method of Periodic Sampling}

The growth curve obtained by the method of periodic sampling (see Materials and Methods) is shown in Fig. 1, in which the number of CFU recovered per spleen is plotted against the time after cell injection at which the spleens were taken. The data used in constructing this figure are presented in Table I. It is apparent from the figure that, after an initial drop in the number of CFU recovered from the spleens of the recipient animals, an exponential increase in this number was observed for the next 7 days. The doubling time of this increase was approximately 25 hours.

It is probable that both the initial decrease in the number of CFU per spleen and the rather large fluctuations in the experimental points were due to an inability to achieve a uniform efficiency of extraction or dispersal of CFU from the irradiated spleens as a function of time.

Effects of Short Interval between Transplantation and Irradiation on Survival of Colony-Forming Ability

The experimental results obtained by using the technique of periodic sampling during the growth of colony-forming cells did not provide a definitive description

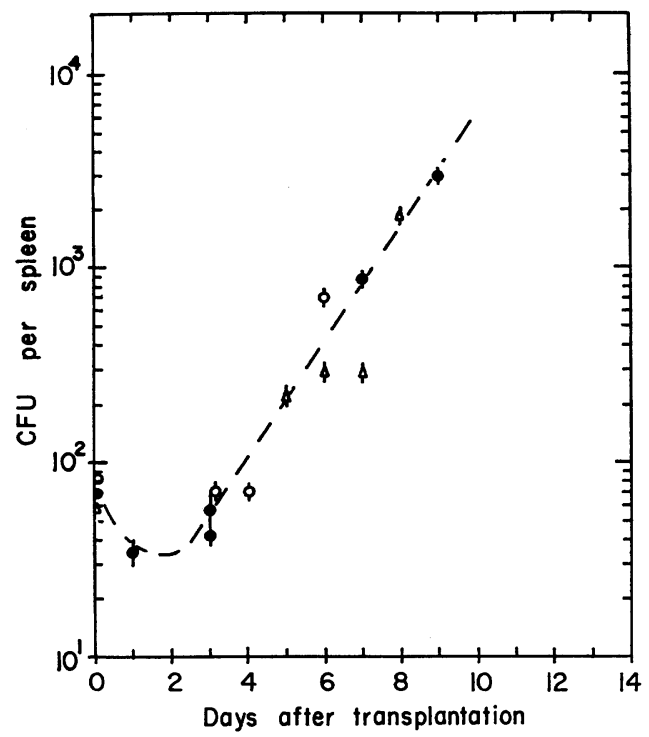

Fig. 1. Growth curve for colony-forming cells derived from (C3H $\times$ C57BL)F1 marrow, obtained by the method of periodic sampling. Points obtained from three separate experiments are shown with their standard errors. 


\section{TABLE I}

Measurement of the Growth Curve for Colony-Forming Cells Derived from $(\mathrm{C} 3 \mathrm{H} \times \mathrm{C} 57 \mathrm{BL}) \mathrm{F} 1$ Marrow by Using the Transplantation Technique

\begin{tabular}{|c|c|c|c|c|}
\hline Experiment & $\begin{array}{c}\text { Day after } \\
\text { transplantation }^{\mathrm{a}}\end{array}$ & $\begin{array}{l}C F U \text { per } 10^{5} \text { cells } \\
\text { recovered from spleen }\end{array}$ & $\begin{array}{c}\text { Cells recovered } \\
\text { per spleen }\left(\times 10^{-7}\right)^{\mathrm{b}}\end{array}$ & $\begin{array}{l}\text { CFU recovered } \\
\text { per spleen }\end{array}$ \\
\hline \multirow[t]{6}{*}{1} & 0 & $0.058 \pm 0.003$ & $12.2 \pm 0.3$ & $71 \pm 4$ \\
\hline & 1 & $0.165 \pm 0.024$ & $2.12 \pm 0.08$ & $35 \pm 5$ \\
\hline & 3 & $0.36 \pm 0.02$ & $1.16 \pm 0.05^{\mathrm{c}}$ & $42 \pm 3$ \\
\hline & 3 & $0.50 \pm 0.10$ & $1.16 \pm 0.05^{\mathrm{c}}$ & $58 \pm 12$ \\
\hline & 7 & $3.15 \pm 0.14$ & $2.78 \pm 0.07$ & $875 \pm 45$ \\
\hline & 9 & $5.18 \pm 0.30$ & $5.84 \pm 0.19$ & $3030 \pm 200$ \\
\hline \multirow[t]{4}{*}{2} & 0 & $0.060 \pm 0.003$ & $14.0 \pm 0.5$ & $84 \pm 5$ \\
\hline & 3 & $0.425 \pm 0.033$ & $1.70 \pm 0.07$ & $72 \pm 6$ \\
\hline & 4 & $0.89 \pm 0.04$ & $0.80 \pm 0.03$ & $71 \pm 4$ \\
\hline & 6 & $1.68 \pm 0.12$ & $4.25 \pm 0.12$ & $715 \pm 64$ \\
\hline \multirow[t]{5}{*}{3} & 0 & $0.046 \pm 0.004$ & $12.7 \pm 0.4$ & $58 \pm 5$ \\
\hline & 5 & $1.92 \pm 0.08$ & $1.14 \pm 0.08$ & $219 \pm 18$ \\
\hline & 6 & $1.41 \pm 0.11$ & $2.10 \pm 0.08$ & $296 \pm 26$ \\
\hline & 7 & $2.78 \pm 0.25$ & $1.04 \pm 0.04$ & $289 \pm 28$ \\
\hline & 8 & $4.60 \pm 0.28$ & $3.98 \pm 0.09$ & $1830 \pm 120$ \\
\hline
\end{tabular}

a Each experimental group consisted of 10 to 15 mice each given $2 \times 10^{6}$ marrow cells.

b Errors shown are standard errors of the mean.

c Same cell suspension.

of the growth curve for colony-forming cells because of the possibility of error introduced by variations in the efficiency of recovery or dispersal of colony-forming cells from the spleens of the test mice. For this reason, the radiobiological technique for measuring cell proliferation was also employed, as this method avoids the necessity of preparing spleen cell suspensions. As was pointed out above (see Materials and Methods), the growth of colony-forming cells may be detected as an increase in survival of colony-forming ability with increasing intervals of time between transplantation and irradiation. This technique was applied first to the study of the behavior of colony-forming cells during the first 2 days after transplantation, for the sampling method had indicated the possibility of a loss of colony-forming cells during this period (Fig. 1).

If the results depicted in Fig. 1 were an accurate reflection of events during the first 2 days after transplantation of colony-forming cells, then fractional survival after a fixed dose of radiation delivered at intervals should decrease during the first day, and then increase in the second day. To examine this question, cells were transplanted into irradiated recipients, and at different times shortly after transplantation their radiation survival was measured. The results obtained are depicted in Fig. 2, which shows the survival ratio-that is, the ratio of the fractional survival after a time interval to the fractional survival shortly ( 2 hours) after trans- 
plantation-as a function of the time interval between transplantation and irradiation for four different doses of radiation. It is apparent from the figure that the results did not agree with expectation, in that the survival ratio increased appreciably during the first day after injection of the cells, reaching a peak value, the magnitude of which was dependent on the radiation dose used. In the case of the largest dose of radiation, 490 rads (curve $d$ ), the peak in survival ratio was followed by an apparent decline, reaching a minimum at 2 days. It was thought unlikely that the average number of colony-forming cells in a proliferating transplant would undergo a transient change of the type shown in Fig. $2 d$, and some other explanation for the result was sought. As was pointed out in Materials and Methods, the interpretation of the survival data as changes in cell number depends on the assumption that only changes in extrapolation number and not changes in $D_{0}$ have occurred. This assumption was tested by obtaining a complete survival curve for colony-forming cells irradiated 22 hours after injection-that is, for a time near the peak shown in Fig. 2. The survival curve obtained is shown in Fig. 3 and is compared with the survival curve obtained for cells irradiated 2 hours

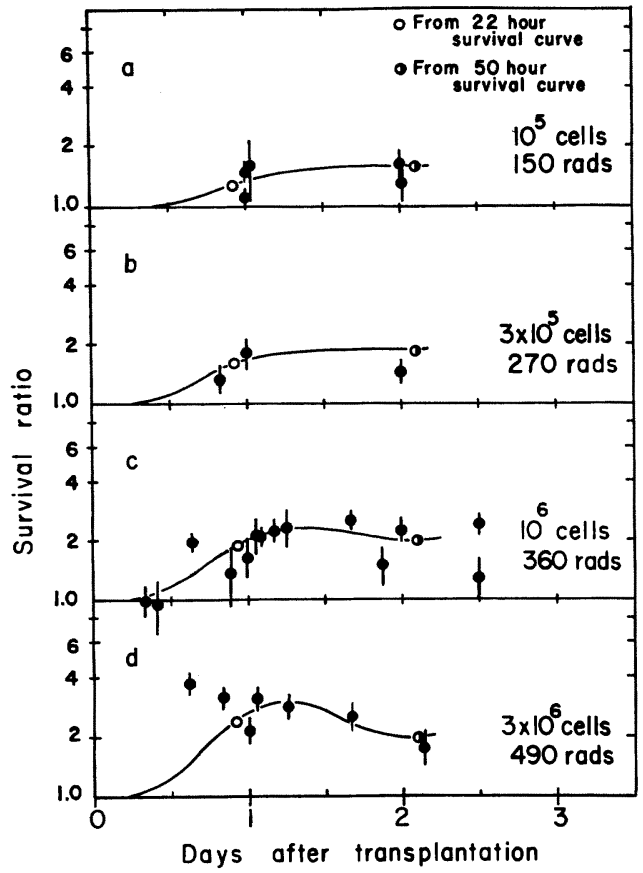

FIG. 2. Survival ratio for colony-forming cells irradiated at various times during the first 2 days after cell transplantation. Experimental points are shown with standard errors (closed circles). The points shown without standard errors were obtained from the 22-hour survival curve of Fig. 3 (open circles) and from the 50-hour survival curve of Fig. 4 (half-closed circles). 
after injection. This latter survival curve has been found to be highly reproducible and is characterized by a $D_{0}$ of 95 rads and an extrapolation number of 1.5. The curve shown without points in Fig. 3 is taken from data previously published ( $y$ ), and, as will be indicated later (see Table II), a point on this curve was obtained in each experiment. The curve drawn through the points in Fig. 3 may be characterized by a $D_{0}$ of 113 rads and an extrapolation number of 1.5. It is apparent from the figure that the experimental points lie well above the 2-hour survival curve, shown without points in the figure. Since the survival curves diverge, the value obtained for the survival ratio will increase with increasing radiation dose. The values of the survival ratio to be expected on the basis of the survival curves shown in Fig. 3 are included as open circles (without errors) in Fig. 2. It is apparent that these points are in good agreement with the other data shown in this figure. Thus, it may be assumed that the initial increase in survival ratio shown in Fig. 2 was due entirely to changes in the slope of the survival curve during the first 2 days after transplantation. The half-filled cirlces (without errors) included in Fig. 2 represent points expected on the basis of the 50-hour survival curve shown in Fig. 4. This survival curve demonstrates that, after 50 hours, the radiation sensitivity of the cells, as measured by the $D_{0}$, had returned to the value of $95 \mathrm{rads}$

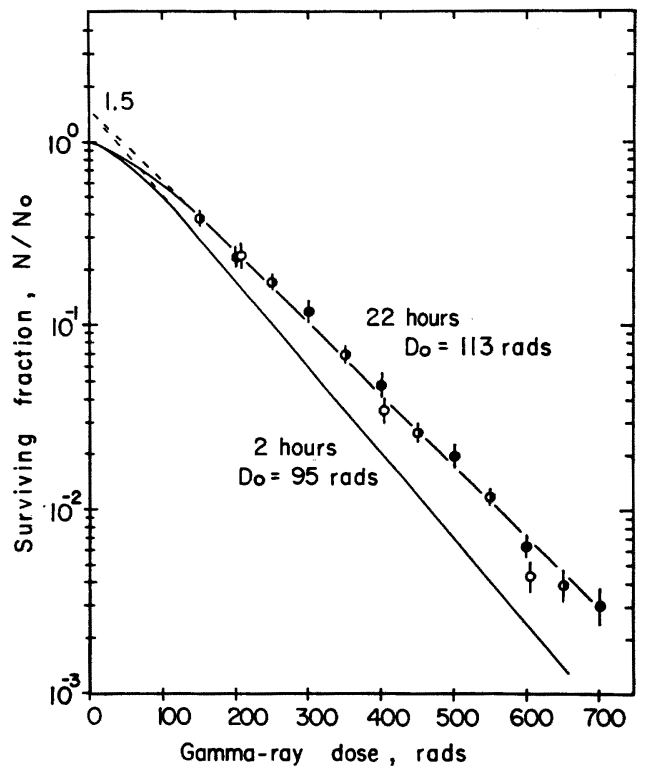

Fig. 3. Survival curves for colony-forming cells irradiated 2 and 22 hours after transplantation. The 2-hour curve $(7)$ is shown without points. For the 22-hour curve, individual points obtained from three separate experiments are shown with their standard errors. The $D_{0}$ and extrapolation number are indicated for each curve. 
characteristic of the 2-hour survival curve. As the points derived from this curve, shown in Fig. 2, are consistent with the experimental data shown in the figure, it is reasonable to assume that the apparent decrease in survival ratio (Fig. $2 c$ and $2 d$ ) results from this change in the slope of the survival curve.

Thus, on the basis of the survival data, when the changes in $D_{0}$ are taken into account, there appears to have been no change in the number of CFU per spleen during the first day after transplantation. This supports the view that the apparent decrease in CFU per spleen observed when the method of periodic sampling was used (Fig. 1) was an artifact of the extraction procedure.

\section{Radiobiological Measurement of the Growth of Colony-Forming Cells}

Before extending the radiobiological method for measuring cell proliferation to later time periods when exponential growth might be expected, it was necessary to ensure that further changes in $D_{0}$ of the survival curve were not occurring. This was done by obtaining survival curves for cells irradiated for 50 hours and 120 hours after transplantation. The results are whown in Fig. 4, and, as in Fig. 3, the survival curve obtained for cells irradiated 2 hours after injection is shown for comparison. It is evident that both the 50-hour and the 120-hour curves lie above the 2-hour curve and are, within the limits of error of the experimental points, parallel to it. Since the $D_{0}$ values derived from the 50-hour and 120 -hour curves

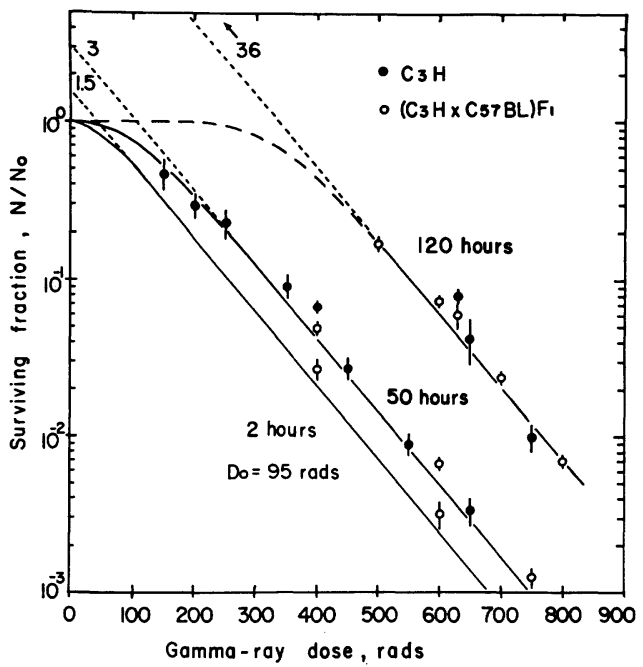

Fig. 4. Gamma-ray survival curves for colony-forming cells irradiated 2, 50, and 120 hours after transplantation. The 2 -hour curve ( 7$)$ is shown without points. For the other curves, the standard errors of the individual points are indicated. The extrapolation numbers obtained by extending the exponential portions of the survival curves back to zero dose (dotted lines) are shown. 
did not differ significantly from the value of 95 rads characteristic of the 2-hour curve, smooth curves characterized by this $D_{0}$ were drawn through the points, as shown. When this was done, the extrapolation numbers obtained were 3 for the 50-hour curve and 36 for the 120-hour curve. The results shown in Fig. 4 indicate that increases in survival occurring at time intervals after transplantation of 50 hours or greater result primarily from changes in extrapolation number.

\section{Construction of a Growth Curve}

On the basis of the above evidence that no significant change in $D_{0}$ occurred for cells irradiated at times later than 50 hours, survival measurements were obtained for various time intervals between cell injection and irradiation and were used to estimate the increase in the number of colony-forming cells by means of the procedure outlined in the Materials and Methods. In such experiments, it is essential that the points measured lie on the exponential portion of the survival curve. This was assured by choosing an appropriate (different) radiation dose for each point, such that the fractional survival of colony-forming ability in each case was below 0.1 (4). The results of a typical experiment are shown in Table II.

The first group shown in Table II was a control group, from which was obtained the number of colony-forming units per $10^{5}$ unirradiated marrow cells (CFU ratio) (7). Each of the subsequent groups was irradiated at a different time interval

TABLE II

Measurement of the Growth Curve for Colony-Forming Cells Derived from C3H Marrow by Using the Radiobiological Technique

\begin{tabular}{|c|c|c|c|c|c|c|c|c|c|}
\hline ड़ & $\begin{array}{l}\text { Dose } D_{1}^{\mathrm{b}} \\
\quad(\text { rads })\end{array}$ & $\begin{array}{c}\text { Cell number } \\
\text { per mouse }\end{array}$ & $\begin{array}{c}\text { Time } \\
\text { interval } \\
\text { (hours) }\end{array}$ & $\begin{array}{c}\text { Dose } \\
D_{2}^{\mathrm{c}} \\
(\text { rads })\end{array}$ & $\begin{array}{c}\text { Mean } \\
\text { colony } \\
\text { number per } \\
\text { spleen at } \\
10 \text { days }\end{array}$ & $\begin{array}{l}\text { CFU } \\
\text { ratio per } \\
10^{5} \text { cells }\end{array}$ & $\begin{array}{c}\text { Fraction } \\
\text { surviving, } \\
\quad N / N_{0}\end{array}$ & $\begin{array}{l}\text { Expected } \\
N / N_{0} \text { for } \\
\text { 2-hour } \\
\text { interval }\end{array}$ & $\begin{array}{l}\text { Survival } \\
\text { ratio }^{\mathrm{d}}\end{array}$ \\
\hline 1 & 900 & $8.0 \times 10^{4}$ & - & 0 & 15.5 & 19.4 & 1.00 & 1.00 & 1.0 \\
\hline 2 & 630 & $1.3 \times 10^{6}$ & 2 & 320 & 14.9 & 1.15 & 0.059 & 0.048 & 1.2 \\
\hline 3 & 700 & $1.3 \times 10^{6}$ & 50 & 400 & 17.3 & 1.33 & 0.069 & 0.020 & 3.4 \\
\hline 4 & 680 & $1.3 \times 10^{6}$ & 72 & 470 & 18.9 & 1.45 & 0.075 & 0.0097 & 7.7 \\
\hline 5 & 650 & $1.3 \times 10^{6}$ & 96 & 550 & 16.1 & 1.24 & 0.064 & 0.0041 & 16 \\
\hline 6 & 620 & $1.3 \times 10^{6}$ & 120 & 630 & 20.0 & 1.54 & 0.079 & 0.0017 & 46 \\
\hline 7 & 590 & $1.3 \times 10^{6}$ & 144 & 710 & 14.0 & 1.08 & 0.056 & 0.00073 & 77 \\
\hline
\end{tabular}

a Each group consisted of 30 to 40 animals.

b $D_{1}$ represents the $\mathrm{X}$-ray dose given to the recipient animals before transplantation of the cells.

c $D_{2}$ represents the $\gamma$-ray dose given to the cells in vivo after transplantation into the $\mathrm{X}$ irradiated recipient animals.

d Survival ratios were obtained by dividing the values listed in column 8 by those listed in column 9. 
after the transplantation of a fixed number of marrow cells, but with doses chosen on the basis of exploratory experiments to yield a fractional survival of colonyforming ability of approximately 0.05 (see column 8 of the table). From these data, the survival ratio was calculated, by using expression 1 of Materials and Methods. The calculation of survival ratio depends on a knowledge of the survival curve for cells irradiated 2 hours after transplantation (see Figs. 3 and 4). As mentioned above, this latter curve has been found to be highly reproducible ( $y)$, but, in practice, a point on this curve was included in each experiment. In the experiment given in detail in Table II, this point was obtained from the data of group 2 of the table. Column 9 of the table, which gives the expected survival after 2 hours, was obtained from the earlier data (7).

From the table, it is evident that the total dose given to the recipient mice (the sum of $D_{1}$ and $D_{2}$ ) increased as the interval between doses increased. This change in total dose was necessary to prevent colony formation from endogenous colonyforming cells derived from the host. Also, although this was not the case for the particular experiment shown in Table II, in most experiments irradiated but uninjected groups were included to ensure that endogenous colony formation was not affecting the results. It should be noted that the transplanted cells received only the radiation dose delivered in $D_{2}$, listed in column 5 of the table.

Growth curves obtained in experiments of this design are shown graphically in

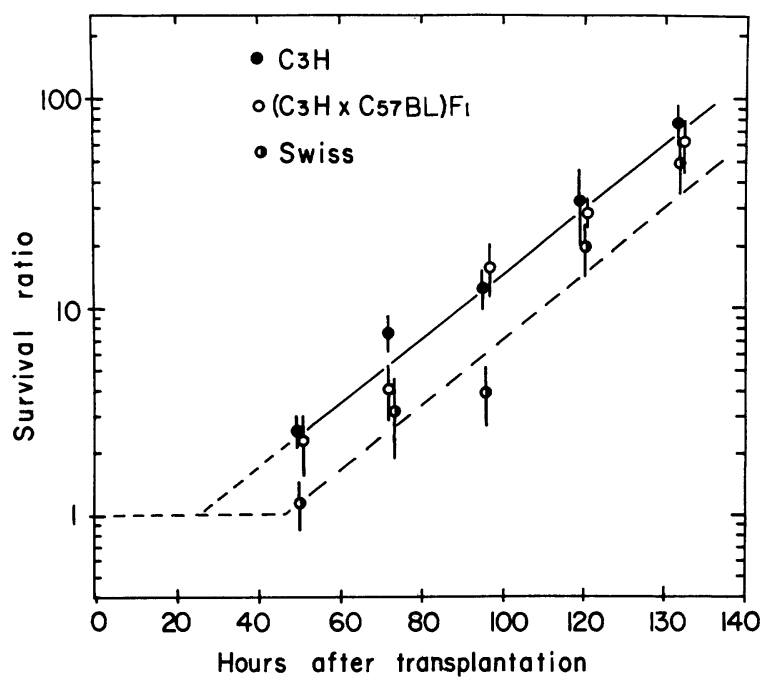

Fig. 5. Survival ratio as a function of the time interval between cell transplantation and irradiation. The points shown for $\mathrm{C} 3 \mathrm{H}$ and $(\mathrm{C} 3 \mathrm{H} \times \mathrm{C} 57 \mathrm{BL}) \mathrm{F} 1$ are the means of several experiments. Standard errors of the individual points are shown. The increase in survival ratio with time is assumed to result from the proliferation of colony-forming cells (see text). 
Fig. 5, in which survival ratio is plotted against time. The marrow suspensions tested were from $\mathrm{C} 3 \mathrm{H}$ mice, $(\mathrm{C} 57 \mathrm{BL} \times \mathrm{C} 3 \mathrm{H}) \mathrm{F} 1$ hybrid mice, and randomly bred Swiss mice. The growth curves for cells from the inbred mice are indistinguishable. Both show an apparent lag period, lasting from 20 to 25 hours, followed by a period of exponential growth which continued for the duration of the experiment. The exponential portion of the curve yields a doubling time for transplanted colonyforming cells of about 20 hours. The growth curve for Swiss cells is similar in form but appears to have a longer lag period, lasting 40 to 50 hours.

\section{DISCUSSION}

Two different methods, one based on periodic sampling and the other on changes in radiation survival, were used to obtain the growth curves for colony-forming cells presented in this paper. Both methods are indirect, for they depend on the measurement of colonies derived from the cells under test, rather than on direct enumeration of the cells. The use of indirect methods is a consequence of the fact that colony-forming cells cannot be distinguished by morphological means from the other cells among which they grow. Fortunately, the two methods complemented each other sufficiently to permit the conclusion that the growth curve for transplanted spleen colony-forming cells derived from normal marrow is characterized by a lag phase of from 1 to 2 days followed by an exponential phase with a doubling time of 20 to 25 hours.

The difficulties encountered with both methods were particularly evident in the measurement of the lag phase of the curve. The results of periodic sampling suggested that there was a loss of colony-forming cells during this phase, whereas the data obtained by the radiobiological method indicated that a transient increase occurred. This latter change was shown to be a consequence of an increase in the $D_{0}$ of the survival curve found when cells were irradiated 22 hours after transplantation. A possible explanation for this change is provided by the experiment of Terasima and Tolmach (10). These authors showed that changes in the form of the radiation survival curve for the colony-forming ability of HeLa S3 cells in tissue culture occur as the cells pass into different phases of the cell cycle. Thus, it is possible that the changes in $D_{0}$ with time observed in our experiments resulted from partial synchronization of the cell population induced by the transplantation procedure. In this way an increased proportion of cells might be in a less radiosensitive portion of the cycle at about 1 day after transplantation, when an increase in $D_{0}$ was found. In this connection, it should be noted that in a previous study no change in the slope of the radiation survival curve was noted during the first 12 hours after transplantation of colony-forming cells derived from marrow (5). Thus, most of the change in radiosensitivity may occur between 12 and 24 hours after transplantation.

Regardless of its mechanism, this change in $D_{0}$ provides an explanation for the 
transient changes in survival shown in Fig. 2, whereas the survival curve shown in Fig. 3 permits a more accurate measurement of the extrapolation number of the survival curve for cells irradiated 22 hours after transplantation. These data give assurance that the number of colony-forming cells in a graft remains relatively constant for the first day after transplantation. On this basis, the changes observed during the same period when the periodic sampling method was used may be considered to be the result of lack of uniformity in the efficiency of extraction or dispersal of $\mathrm{CFU}$ from splenic tissue at different times after transplantation.

Both the periodic sampling and radiobiological techniques indicate an exponential increase in colony-forming cells for times longer than 50 hours after transplantation, but the sampling technique yielded a longer doubling time ( 25 hours) than the radiobiological technique (20 hours). This discrepancy may also result from the limitations of the methods used. For example, failure to obtain uniform efficiency of extraction or dispersal of CFU may be the cause of the wide spread in the data obtained by the sampling method. Further, the radiobiological method depends on the assumption (see Materials and Methods) that the $D_{0}$ of the survival curve is a constant. Although the 120-hour survival curve shown in Fig. 4 supports this assumption, the exponential portion of this survival curve could be extended over only slightly more than one decade of survival and thus may be less reliable than the other survival curves shown. Small changes in the $D_{0}$ at such late times could cause relatively large errors in the estimated cellular multiplicity. A similar explanation may account for the discrepancy between the doubling times found in the present experiments and the doubling time of over 30 hours for endogenous colony-forming cells from spleen observed in a previous series of experiments (1). An attempt to resolve this discrepancy by obtaining more complete survival curves for colony-forming cells from spleen is at present under way.

In spite of the technical difficulties discussed above, the form of the growth curve for colony-forming cells appears to be established in sufficient detail to suggest conclusions based on it. In particular, the fact that in the present experiments no increase in the number of colony-forming cells was detected during the first day after transplantation leads to the conclusion that the early repair of radiation damage to colony-forming cells observed in previous experiments (5) during this period of time was in fact the result of intracellular recovery processes of the type described by Elkind and Sutton (4). Thus, it appears that the initial maximum in early repair observed 5 hours after irradiation of transplanted colony-forming cells $(5)$ was due to an intracellular process of repair of sublethal damage which took place in irradiated colony-forming cells. The fact that a rapid phase of repair of radiation damage, based on measurements of the acutely lethal dose for mice exposed to fractionated doses of total-body irradiation, also appears to be completed within 4 to 6 hours after irradiation $(13,14)$ provides additional support for the view presented in the Introduction that colony-forming cells form an important part of 
the cellular basis for repair of damage induced in the hemopoietic system by wholebody irradiation.

The recent report of Bernstein (15) that congenitally anemic mice with mutant genes at the $\mathrm{W}$ locus are particularly susceptible to the lethal effects of radiation has provided an opportunity to test further the hypothesis that colony-forming cells are important in recovery after irradiation. If the hypothesis is valid, the radiosensitive animals should be found to be deficient in colony-forming cells. The results of preliminary experiments (16) fulfill this prediction. Thus, these experiments provide another example of the correlation between colony-forming ability of hemopoietic cells and the sensitivity of the whole animal to the acutely lethal effects of whole-body irradiation.

\section{SUMMARY}

1. The kinetics of proliferation of spleen colony-forming cells derived from transplanted marrow cells have been studied by means of two different methods. The results of these experiments indicate that transplanted colony-forming cells do not proliferate in heavily irradiated recipients during the first day after transplantation, but that after this initial lag phase they increase in number in an exponential fashion for at least the next 7 days, with a doubling time of 20 to 25 hours.

2. A transient increase in the $D_{0}$ of the survival curve for transplanted colonyforming cells derived from marrow was observed during the posttransplantation lag phase. This increase was maximal approximately 1 day after transplantation.

\section{ACKNOWLEDGMENTS}

We are grateful for the excellent technical assistance of Miss Rosemary Wyncoll, Mrs. Pat McRae, Miss Clair McCausland, and Mr. James Hicks. We also wish to thank Dr. L. Siminovitch for his helpful criticisms during the preparations of the manuscript.

RECEIVED: November 11, 1963

\section{REFERENCES}

1. J. E. Till and E. A. McCulloch, Repair processes in irradiated mouse hematopoietic tissue. Ann. N. Y. Acad. Sci. 114, 115-125 (1964).

2. L. Siminovitch, E. A. McCulloch, and J. E. Till, The distribution of colony-forming cells among spleen colonies. J. Cellular Comp. Physiol. 62, 327-336 (1963).

3. E. A. McCulloch, Les clones de cellules hématopoiétiques in vivo. Rev. Franc. Etudes Clin. Biol. 8, 15-19 (1963).

4. M. M. ELKind and H. Sutton, Radiation response of mammalian cells grown in culture. I. Repair of X-ray damage in surviving Chinese hamster cells. Radiation Res. 13, 556-593 (1960).

5. J. E. Till and E. A. McCulloch, Early repair processes in marrow cells irradiated and proliferating in vivo. Radiation Res. 18, 96-105 (1963).

6. E. A. McCulloch and J. E. Tilu, Repression of colony-forming ability of C57BL hematopoietic cells transplanted into non-isologous hosts. J. Cellular Comp. Physiol. 61, 301308 (1963). 
7. E. A. McCulloch and J. E. Till, The sensitivity of cells from normal mouse bone marrow to gamma radiation in vitro and in vivo. Radiation Res. 16, 822-832 (1962).

8. K. Scharf and R. M. LeE, Investigation of the spectrophotometric method of measuring the ferric ion yield in the ferrous sulfate dosimeter. Radiation Res. 16, 115-124 (1962).

9. J. E. Till and E. A. McCulloch, A direct measurement of the radiation sensitivity of normal mouse bone marrow cells. Radiation Res. 14, 213-222 (1961).

10. T. Terasima and L. J. Tolmach, Variations in several responses of HeLa cells to X-irradiation during the division cycle. Biophys. J. 3, 11-33 (1963).

11. M. M. Elkind, H. Sutton, and W. B. Moses, Postirradiation survival kinetics of mammalian cells grown in culture. J. Cellular Comp. Physiol. 58, Suppl. 1, 113-134 (1961).

12. S. E. LURIA and R. LATARJET, Ultraviolet irradiation of bacteriophage during intracellular growth. J. Bacteriol. 53, 149-163 (1947).

13. J. F. Fowler, D. K. Bewley, R. L. Morgan, J. A. Silvester, T. Alper, and S. Hornsey, Dose-effect relationships for radiation damage to organized tissues. Nature 199, 253255 (1963).

14. J. A. H. Brown, M. J. Corp, and R. H. Mole, The effect of dose-rate and fractionation on acute mortality in X-irradiated mice. Part II. Intern. J. Radiation Biol. 5, 369-377 (1962).

15. S. E. Bernstein, Acute radiosensitivity in mice of differing $\mathrm{W}$ genotype. Science 137, 428-429 (1962).

16. E. A. McCulloch, L. Siminovitch, and J. E. Till, Spleen-colony formation in anaemic mice of genotype $W^{v}$. Science in press. 